\title{
Abordagem Terapêutica da Obesidade na Síndrome de Prader-Willi
}

\begin{abstract}
RESUMO
A Síndrome de Prader-Willi (SPW) é uma doença complexa, multissistêmica, caracterizada por hipotonia, retardo mental, características dismórficas, hiperfagia e compulsão alimentar devido à disfunção hipotalâmica. SPW ocorre pela perda de função de genes localizados no cromossomo 15q11-13, região que sofre imprinting genômico. Obesidade é a principal causa de morbidade e mortalidade entre pacientes com SPW. O objetivo desta revisão é analisar as opções terapêuticas disponíveis para o tratamento da obesidade na SPW, incluindo a terapia farmacológica e o tratamento cirúrgico. (Arq Bras Endocrinol Metab 2007;51/6:913-919)

Descritores: Obesidade; Síndrome de Prader-Willi; Tratamento farmacológico; Cirurgia bariátrica; Topiramato

ABSTRACT

Therapeutical Approach of Obesity in Prader-Willi Syndrome. Prader-Willi Syndrome (PWS) is a multisystemic genetic disease characterized by hypotonia, mental retardation, characteristic facial appearance, hyperphagia, and compulsive eating due to hypothalamic dysfunction. PWS is caused by loss of function of genes located in chromosome 15q11-q13, an area subject to genomic imprinting. Obesity is a major cause of increased morbidity and mortality among patients with PWS. The objective of this study was to analyze the therapeutic options available for the treatment of the obesity in PWS including pharmacological and surgical strategies. (Arq Bras Endocrinol Metab 2007;51/6:913-919)
\end{abstract}

Keywords: Obesity; Prader-Willi syndrome; Pharmacological treatment; Bariatric surgery; Topiramate

E M 1956, Prader, LabHart E Willi descreveram uma condição com fenótipo constituído por baixa estatura, obesidade, retardo mental, criptorquidia e hipotonia na infância, denominada Síndrome de Prader-Willi (1). A Síndrome de Prader-Willi (SPW) é uma doença complexa, multissistêmica, caracterizada por hipotonia, retardo mental, características dismórficas e disfunção endócrina hipotalâmica (2). A prevalência da SPW é de 1 para cada 15.000 nascimentos, variando de 1:10.000 a 1:16.000 e acometendo igualmente ambos os sexos (3). Em relação ao tratamento da SPW, um interesse crescente tem sido demonstrado no manejo de condições associadas a um prognóstico desfavorável, sobretudo a obesidade. Os principais fatores desencadeantes da mortalidade na SPW estão relacionados à obesidade, o que torna o controle desta condição fundamental para a melhora da sobrevida destes pacientes (4). O objetivo desta revisão é analisar as opções terapêuticas disponíveis para o tratamento da obesidade na SPW.

\section{revisão}

\author{
Daniel F. de Carvalho \\ Cíntia Cercato \\ MAdSON Q. ALMEIDA \\ MARCIO C. MANCINI \\ Alfredo Halpern
}

Grupo de Obesidade e Síndrome Metabólica, Divisão de Endocrinologia, Hospital das Clínicas, Faculdade de Medicina da Universidade de São Paulo, SP.

Recebido em 23/1 1/06 Revisado em 14/03/07 Aceito em 13/04/07 


\section{ASPECTOS DIAGNÓSTICOS}

A principal alteração genética associada à SPW é a deleção do segmento 15 q11-13 de origem paterna, que está presente em $70-75 \%$ dos pacientes. Outras anormalidades têm sido relacionadas a SPW: dissomia materna do cromossomo 15 (ambos os cromossomos 15 são de origem materna) em $25 \%$ dos casos; mutações epigenéticas (2-5\%) e translocações (1\%) (5). Como o segmento $15 \mathrm{qll}-13$ contém genes que sofrem imprinting materno (alelo materno inativo), a deleção do segmento de origem paterna ou duplicação do cromossomo materno ocasionam a perda da função de genes paternalmente expressos contidos neste locus.

O diagnóstico da SPW baseia-se em critérios clínicos e o diagnóstico definitivo pode ser confirmado através da análise genética (6). Como nem sempre o estudo genético está disponível na prática médica, a utilização de critérios clínicos é de grande utilidade. Em 1993, Holm e cols. propuseram critérios clínicos maiores e menores para o diagnóstico da síndrome (tabela 1) (7), com uma sensibilidade que varia entre os critérios maiores de 49\% (características faciais) a 98\% (retardo mental) e, para os critérios menores, de 37\% (apnéia do sono) a 93\% (defeitos na articulação de palavras). Em 2001, foi proposto por Gunay-Aygun e cols. uma nova abordagem desses critérios, com a divisão de acordo com as faixas etárias em que podem se manifestar, que parecem contribuir na triagem de indivíduos que deveriam realizar o teste genético, quando disponível, pela alta probabilidade de serem portadores da SPW (tabela 2) (8).

As alterações encontradas na SPW podem ser divididas em três categorias principais: alterações estruturais, comportamentais e intelectuais.

\section{Alterações estruturais}

Baixa estatura está presente em até 90\% dos indivíduos afetados (3). A diminuição da massa magra e o aumento da massa gorda contribuem para a hipotonia e a obesidade (9). Além disso, a relação massa gorda/ massa magra é maior nos pacientes com SPW quando comparada a indivíduos de peso normal e obesos não portadores da SPW (10). Acredita-se que essas alterações estruturais estejam relacionadas à deficiência de hormônio do crescimento $(\mathrm{GH})$, também característica da síndrome (11). Hipogonadismo hipogonadotrófico hipotalâmico pode estar presente na SPW, levando a um quadro de retardo puberal e infertilidade (12). Pacientes com SPW apresentam também diminuição da mineralização óssea, fato que explica a incidência aumentada de osteoporose associada à síndrome (10).

\section{Alterações comportamentais}

Os pacientes com SPW apresentam comportamento impulsivo e compulsivo, que pode se expressar através de episódios de auto-escoriações, distúrbios ansiosos, como transtorno obsessivo-compulsivo e hábito alimentar compulsivo, o que constitui um fator determinante na progressão da obesidade. Após o período da adolescência, podem apresentar, ainda, transtornos afetivos com psicoses recorrentes, bem como quadros com características catatônicas, que ocorrem provavelmente por disfunção gabaérgica (13).

\section{Alterações intelectuais}

Graus variáveis de retardo mental, dificuldade de aprendizado e menores médias de coeficiente de inteligência têm sido observados em indivíduos com SPW (14).

\section{OBESIDADE NA SPW}

A SPW é reconhecidamente a síndrome genética mais comumente associada à obesidade. A obesidade constitui a principal causa de aumento de morbidade e mortalidade prematura nos pacientes com SPW, que apresentam risco elevado de desenvolver hipertensão arterial, diabetes mellitus, insuficiência respiratória e insuficiência cardíaca (15). A obesidade na SPW é conseqüência da diminuição de saciedade associada ao comportamento alimentar compulsivo, ambos decorrentes de disfunção hipotalâmica (16). Além disso, distúrbios endócrinos e metabólicos, tais como deficiência de GH e alteração da composição corporal, parecem ter um papel importante na patogênese da obesidade na SPW.

O apetite humano é regulado por diversas vias complexas, que envolvem desde estruturas centrais até órgãos periféricos, com numerosas intersecções entre seus ramos. Sabe-se que, centralmente, o apetite tem sua modulação realizada no hipotálamo, sobretudo nos núcleos paraventriculares. Perifericamente, vários hormônios e peptídeos agem em duas vias principais que são responsáveis pelo equilíbrio entre a fome e a saciedade, a via orexigênica, que estimula o apetite, e a via anorexigênica, que o inibe. A complexidade da fisiologia da regulação do apetite e da saciedade, além da impossibilidade de intervenção em pontos isolados e específicos dessa cascata, dificultam o tratamento farmacológico da obesidade, particularmente na SPW.

O comportamento alimentar compulsivo pode ser explicado pelo fato de os portadores da SPW não atingirem um platô de saciedade na medida em que a ingestão progride, apresentando um padrão linear de 
Tabela 1. Critérios clínicos diagnósticos da SPW (7).

Critérios maiores (1 ponto)
Hipotonia neonatal
Dificuldade de alimentação na infância ou retardo do desenvolvimento neuro-psicomotor
Ganho excessivo de peso a partir de 1 ano e 6 meses
Hipogonadismo
Retardo mental, problemas de aprendizado
Hiperfagia, obsessão por comida
Características faciais (olhos amendoados, lábio superior pouco espesso)
Deleção do locus $15 q 11-q 13$ em análise citogenética
Critérios menores (0,5 ponto)
Diminuição dos movimentos fetais
Problemas de comportamento (5 destes: fúria, acessos de violência, obsessivo-compulsivo,
argumentativo, rígido, possessivo, teimoso, mentiroso, cleptomania)
Apnéia do sono
Baixa estatura
Cabelos, olhos e pele mais claros
Mãos e pés pequenos
Mãos estreitas
Miopia
Saliva viscosa
Defeito na articulação de palavras
Lesões de pele (auto-agressão)

Critérios diagnósticos: 5 pontos, sendo 4 de critérios maiores em pacientes com idade $\leq 3$ anos; 8 pontos, sendo 5 de critérios maiores em pacientes com idade $>3$ anos.

Tabela 2. Novos critérios sugeridos para solicitação da análise genética para Síndrome de Prader-Willi.

\begin{tabular}{|c|c|}
\hline Idade & Critérios \\
\hline 0 a 2 anos & 1. Hipotonia com má sucção \\
\hline 2 a 6 anos & $\begin{array}{l}\text { 1. Hipotonia com história de má sucção } \\
\text { 2. Atraso global do desenvolvimento }\end{array}$ \\
\hline 6 a 12 anos & $\begin{array}{l}\text { 1. História de hipotonia com história de má sucção (a hipotonia } \\
\text { geralmente persiste) } \\
\text { 2. Atraso global do desenvolvimento } \\
\text { 3. Excessiva ingesta alimentar (hiperfagia, obsessão por } \\
\text { alimento) com obesidade central resultante se não controlada }\end{array}$ \\
\hline Maiores de 12 anos & $\begin{array}{l}\text { 1. Funções cognitivas prejudicadas; geralmente retardo mental } \\
\text { moderado } \\
\text { 2. Excessiva ingesta alimentar (hiperfagia, obsessão por } \\
\text { alimento) com obesidade central resultante se não controlada } \\
\text { 3. Hipogonadismo hipogonadotrófico e/ou problemas } \\
\text { comportamentais típicos (incluindo transtornos obsessivo- } \\
\text { compulsivos e temperamento tântrico) }\end{array}$ \\
\hline
\end{tabular}

A análise genética está indicada caso os pacientes com suspeita diagnóstica de Prader-Willi apresentem todos os critérios correspondentes à sua faixa etária.

ingestão em comparação com indivíduos normais (17). Um estudo realizado em 2005 por Hinton e cols. mostrou que pacientes com SPW mantêm atividade presente no centro regulador da fome mesmo após ingestão de refeições de até $1.200 \mathrm{kcal}$, quando comparado com indivíduos não obesos e não portadores da SPW. Essa atividade foi medida por PET - Positron Emission Tomography - e parece estar relacionada ao padrão linear de ingestão alimentar (18). Ocorre, ainda, atraso na ativação do centro da saciedade, avaliado por Shapira e col., através de imagens de ressonância nuclear magnética funcional, 
de até 14 minutos após ingestão de $75 \mathrm{~g}$ de glicose em relação a indivíduos normais, e de até 9 minutos em relação a obesos não portadores da síndrome (19). Também estão presentes nesses indivíduos alterações celulares hipotalâmicas, tais como redução significativa no volume do núcleo paraventricular e em seu número de células, bem como a redução de $43 \%$ dos neurônios que expressam ocitocina, um dos principais peptídeos anorexigênicos (20).

Outro fator importante na gênese da obesidade da SPW é a deficiência de hormônio de crescimento (DGH), o que determina um menor crescimento estatural e alterações metabólicas desfavoráveis, além do desbalanço entre massa magra e massa gorda corporal (11).

Recentemente foi demonstrado que portadores da SPW apresentam níveis elevados de ghrelina, peptídeo orexigênico produzido pelas células do fundo gástrico, que atua como secretagogo endógeno de GH. O aumento da ghrelina parece desempenhar um papel crucial na fisiopatologia das alterações fenotípicas da SPW. A ghrelina elevada estimula populações neuronais que expressam neuropeptídeo Y (NPY) do núcleo arqueado do hipotálamo. O NPY, por sua vez, leva a três conseqüências: redução do nível de $\mathrm{GH}$, diminuição da função reprodutora e hiperfagia (21).

A causa para elevação dos níveis plasmáticos de ghrelina na SPW ainda é desconhecida. Um estudo que comparou os níveis de ghrelina entre 5 pacientes com SPW e 5 controles normais ajustados para idade, sexo e IMC, observou que o nível de ghrelina era 3 a 4 vezes maior nos pacientes com SPW (22). Sabe-se que a somatostatina é um peptídeo que inibe a secreção de ghrelina pelas células do fundo gástrico; entretanto, a administração de somatostatina a indivíduos com SPW não se mostrou eficaz na redução da ingestão alimentar, embora tenha reduzido os níveis séricos de ghrelina e de neuropeptídeo $\mathrm{Y}$ e causado hiperglicemia pós-prandial nesses pacientes (23).

A associação desses fatores torna o controle da obesidade na SPW um desafio. A obesidade mórbida resultante, além de aumentar a morbimortalidade, torna extremamente difícil o controle dos outros comportamentos compulsivos, tais como agressividade e automutilação.

\section{TRATAMENTO}

As opções terapêuticas para a abordagem da obesidade na SPW são: medidas dietéticas, tratamento farmacológico e abordagem cirúrgica.

\section{Medidas dietéticas}

Restrição dietética com a finalidade de diminuir as calorias ingeridas pelos pacientes é ineficaz na redução de peso e manutenção do peso perdido quando realizada isoladamente, pois, obviamente, os portadores de SPW têm sua adesão prejudicada pelo mecanismo orexigênico de origem hipotalâmica presente na síndrome, que é associado ao comportamento compulsivo.

\section{Tratamento farmacológico}

\section{Hormônio de crescimento $(G H)$}

$\mathrm{O}$ GH tem um papel benéfico no crescimento longitudinal a longo prazo quando administrado em idade adequada em pacientes com SPW por aumentar a velocidade de crescimento e a estatura final (24).

Ensaios clínicos controlados têm demonstrado efeito favorável da reposição de GH na melhora da composição corporal, com redução da massa gorda e aumento da massa magra, quando administrado juntamente a orientação nutricional adequada $(24,25)$. A terapia com $\mathrm{GH}$, entretanto, não parece ter efeito significativo no controle dos níveis séricos de substâncias envolvidas na regulação do apetite, que estão classicamente alteradas na SPW e que contribuem de maneira decisiva para a hiperfagia apresentada por esses pacientes (26).

Um estudo que avaliou o papel da administração de GH em relação ao metabolismo de carboidratos em 17 portadores de SPW observou que houve melhora da intolerância à glicose, além de normalização dos níveis séricos de insulina e regressão da resistência à insulina. Além disso, a glicemia de jejum, os níveis de hemoglobina glicada e de triglicérides foram normais e não se alteraram durante todo o estudo. Houve, ainda, normalização da porcentagem de massa gorda, que era de, em média, 39,5\% antes da terapia proposta e atingiu $28,3 \%$ após os 3 anos de tratamento. O retardo na secreção de insulina não foi alterado com os 3 anos de administração do hormônio (27).

Os efeitos colaterais observados pela administração de GH são os mesmos observados na reposição em pacientes com DGH primária ou decorrente de outras doenças. Acreditava-se que o rápido crescimento promovido pelo GH poderia piorar a escoliose já observada nos portadores de SPW. Contudo, não foi observada piora significativa da escoliose em estudos com seguimento de doze meses $(24,25)$.

Existem alguns relatos de caso de morte súbita em pacientes portadores de SPW após início da terapia de reposição com GH (28-31). Em razão disso, foram realizados estudos com a finalidade de elucidar o 
possível mecanismo e uma das hipóteses levantadas foi que o GH leva a certo grau de obstrução das vias aéreas, podendo piorar a apnéia do sono agravando o quadro respiratório. Com base nisso, foi proposto por Eiholzer e col. que, antes do início da terapia com $\mathrm{GH}$, esses indivíduos devem realizar poli-sonografia e a exame otorrinolaringológico completo (32).

\section{Topiramato}

O topiramato é um anticonvulsivante de nova geração cujos mecanismos de ação parecem ser atividade agonista gabaérgica, antagonismo do sistema glutamatérgico e modulação de canais de sódio voltagem-dependente. Recentemente, vem sendo estudado como possível droga antiobesidade, especialmente em casos de compulsão alimentar ou binge-eating (33).

Evidências de disfunção hipotalâmica na SPW, como diminuição da atividade gabaérgica, podem explicar o efeito benéfico da administração de anti-convulsivantes nesses pacientes. $\mathrm{O}$ aumento da atividade gabaérgica promovido pelas drogas anti-epilépticas pode estar relacionado ao aumento de peso observado com o uso dessa classe de medicação. Por outro lado, a atividade antagonista glutamatérgica do topiramato é o mecanismo proposto que poderia explicar a ação desta droga na promoção de perda de peso ou diminuição do ganho de peso nos pacientes com SPW (34).

Em 2003, Smathers e col. (35) avaliaram o efeito do topiramato (100 a $600 \mathrm{mg} /$ dia de dose máxima tolerada) em 7 pacientes com SPW. Os pacientes apresentaram melhora importante do comportamento compulsivo e agressividade. O efeito do topiramato na perda de peso não foi tão evidente, mas todos os pacientes tiveram estabilização do ganho ponderal progressivo. Em outro estudo, essa droga não se mostrou eficaz na redução da ingestão calórica ou do apetite quando administrada por 8 semanas (36). Porém, outros estudos demonstraram efeito benéfico do topiramato em reduzir lesões provocadas por comportamento de automutilação em pacientes com SPW $(36,37)$.

A experiência de nosso grupo mostrou que a administração de topiramato na dose de $100 \mathrm{mg} /$ dia, por 20 semanas, em 2 adultos com SPW resultou em perda de $4 \%$ e $5 \%$ do peso corporal nesses pacientes (38). No entanto, poucos são os dados da literatura até o momento com o uso do topiramato na SPW, sendo necessários mais estudos para estabelecer seu papel no tratamento da obesidade associada à síndrome.

\section{Drogas antiobesidade}

Existe um certo receio no uso de drogas antiobesidade na SPW por poderem, supostamente, piorar a longo prazo o comportamento agressivo apresentado por esses pacientes, sem uma melhora significativa e permanente do peso corporal.

Não há relatos na literatura do uso de sibutramina ou orlistate nestes pacientes. Por outro lado, existem alguns relatos do uso de mazindol em pacientes com SPW (39-41).

O mazindol é uma droga catecolaminérgica cuja ação envolve aumento da ação da dopamina e da norepinefrina em receptores pós-sinápticos, resultando em efeito anorexigênico por ação hipotalâmica. Mostrou-se eficaz na redução do ganho de peso a curto prazo em um estudo realizado no Japão com dois portadores da síndrome, aos quais mazindol foi administrado na dose de 1 a $2 \mathrm{mg} / \mathrm{dia}$, em uma ou duas doses diárias, por 24 semanas, tendo havido redução dos valores do IMC de 36,0 para $29,7 \mathrm{~kg} / \mathrm{m}^{2}$ e de 43,7 para $37,3 \mathrm{~kg} / \mathrm{m}^{2}$, sem efeitos colaterais significativos (40). Um relato de caso sugeriu, ainda, que o mazindol na dose de $1 \mathrm{mg} /$ dia foi efetivo não só na redução de peso de até $5 \%$, mas também na melhora dos níveis de hemoglobina glicada em um paciente diabético portador da SPW (41). Ainda são necessários estudos com essa classe de medicamentos em portadores de SPW por períodos mais prolongados.

A fluoxetina, um medicamento da classe dos inibidores seletivos da recaptação de serotonina, tem sido utilizada como coadjuvante no tratamento da obesidade por diminuir episódios de compulsão alimentar, diminuindo a ingestão calórica em pacientes com esse padrão alimentar (42). Por essa razão, seu uso na SPW pode ser benéfico não só na redução de peso, mas também na melhora do comportamento compulsivo.

Dech \& Budow descreveram caso de uma portadora de SPW que apresentou grande melhora no controle do peso e moderada melhora na tricotilomania e tricofagia após introdução de fluoxetina (43). Outros relatos de caso mostraram que a fluoxetina, além de auxiliar no controle do peso, reduziu o comportamento de automutilação (44-46). Porém, outros autores não conseguiram demonstrar tais benefícios com o uso de fluoxetina $(47,48)$.

Nosso grupo descreveu o caso de um paciente portador de SPW de 25 anos, do sexo masculino, que foi tratado com fluoxetina na dose de $40 \mathrm{mg} / \mathrm{dia}$ por 6 meses associada à dieta hipocalórica, e obteve como resultado uma perda de $17 \%$ (20 kg) de peso corporal, melhora da apnéia do sono e do comportamento compulsivo (49), porém este efeito não se manteve a longo prazo. 
Mais estudos são necessários para que maiores conclusões possam ser obtidas sobre o papel dessa droga no tratamento de pacientes com SPW.

\section{TRATAMENTO CIRÚRGICO}

Restrição dietética, sozinha ou combinada com drogas anoréticas, tem-se mostrado ineficaz para induzir perda de peso prolongada. Cirurgia bariátrica tem sido uma opção para estes pacientes.

Muitos procedimentos cirúrgicos têm sido descritos em portadores da SPW. A gastroplastia vertical foi realizada em uma paciente com SPW, tendo ocorrido ruptura parcial da linha de grampeamento no 11 O mês pós-operatório (50). O comportamento alimentar da paciente pode ter sido a causa para a falência da gastroplastia. A paciente não conseguia parar de comer, devido ao apetite insaciável associado à SPW, o que contribuiu para um aumento da pressão na bolsa gástrica, resultando na sua ruptura.

De acordo com uma revisão recente que fizemos da literatura, a derivação biliopancreática (DBP), procedimento malabsortivo, parece ser uma abordagem cirúrgica mais adequada para esses pacientes (51). Nesta revisão, relatamos a cirurgia de DBP pela técnica de Scopinaro em dois portadores da SPW, que foi eficaz na redução de peso e manutenção prolongada, com redução do IMC de $52 \mathrm{~kg} / \mathrm{m}^{2}$ para $36,7 \mathrm{~kg} / \mathrm{m}^{2} \mathrm{em}$ um homem de 27 anos, acompanhado por 12 meses, e de $64 \mathrm{~kg} / \mathrm{m}^{2}$ para $48,8 \mathrm{~kg} / \mathrm{m}^{2}$ em uma paciente do sexo feminino, de 20 anos, seguida por 28 meses, com melhora marcante da função respiratória, além de melhora do comportamento agressivo, resultando em melhor qualidade de vida (51).

É preciso, porém, estar vigilante em relação às complicações do tratamento cirúrgico, que podem ocasionar a médio e longo prazo desnutrição protéicocalórica e ou vitamínico-mineral, tais como anemia, hipoproteinemia, diminuição da densidade óssea, além de diarréia associada à alimentação (52). Entretanto, essas condições podem, em sua maioria, ser corrigidas através da reposição de vitaminas e minerais e de uma alimentação com conteúdo protéico adequado.

\section{CONCLUSÃO}

Tendo em vista as principais características da obesidade da SPW, associadas aos outros achados comportamentais da síndrome, percebe-se que o tratamento da obesidade nesses pacientes é um verdadeiro desafio. A reposição de GH mostrou-se eficaz e está bem estabelecida na melhora da composição corporal nesses indivíduos. Por sua vez, a experiência com o uso de drogas antiobesidade na prática diária tem aumentado, ressaltando-se a necessidade de mais estudos para estabelecer seu uso, segurança e indicações no manejo desses pacientes. O tratamento cirúrgico temse estabelecido como efetivo por não depender de medidas dietéticas tão restritas no pós-operatório, porém devem ser lembradas as principais complicações a longo prazo e tomadas as devidas condutas para evitá-las.

\section{REFERÊNCIAS}

1. Prader A, Labhart A, Willi $H$. Eins Syndrom von Adipositas, Kleinwuchs, Kryptochismus und Oligophrenie nach myotoniertgem Zustand im Neugeborenalter. Schweiz Med Wochenschr 1956;86:1260-1.

2. Butler MG, Thompson T. Prader-Willi syndrome: clinical and genetic findings. Endocrinologist 2000;10:3S-16S.

3. Cassidy SB. Prader-Willi syndrome. J Med Genet 1997; 34:917-23.

4. Einfeld SL, Kavanagh SJ, Smith A, Evans EJ, Tonge BJ, Taffe J. Mortality in Prader-Willi syndrome. Am J Ment Retard 2006;111(3):193-8.

5. Nicholls RD, Saitoh S, Horsthemke B. Genetic abnormalities in Prader-Willi syndrome and lessons from mouse models. Acta Paediatr Suppl 1999;88:99-104.

6. Bittel DC, Butler MG. Prader-Willi syndrome: clinical genetics, cytogenetics and molecular biology. Expert Rev Mol Med 2005;7(14):1-20.

7. Holm VA, Cassidy SB, Butler MG, Hanchett JM, Greenswag LR, Whitman BY, et al. Prader-Willi syndrome: consensus diagnostic criteria. Pediatrics 1993;91:398-402.

8. Gunay-Aygun M, Schwartz S, Heeger S, O'Riordan MA, Cassidy SB. The changing purpose of Prader-Willi syndrome: clinical diagnostic criteria and proposed revised criteria. Pediatrics 2001;108(5):E92.

9. Eiholzer U, Blum WF, Molinari L. Body fat determined by skinfold measurements is elevated despite underweight in infants with Prader-Willi syndrome. J Pediatrics 1999;134:222-5.

10. Brambilla $P$, Bosio $L$, Manzoni $P$, Pietrobelli A, Beccaria L, Chiumello G. Peculiar body composition in patients with Prader-Labhart-Willi syndrome. Am J Clin Nutr 1997;65:1369-74.

11. Burman $P$, Ritzen EM, Lindgren AC. Endocrine dysfunction in Prader-Willi syndrome: a review with special reference to $\mathrm{GH}$. Endocr Rev 2001;22:787-99.

12. Eiholzer U, I'Allemand D, Rousson V, Schlumpf M, Gasser T, Girard J, et al. Hypothalamic and gonadal components of hypogonadism in boys with Prader-Labhart-Willi syndrome. J Clin Endocrinol Metab 2006;91(3):892-8.

13. Verhoeven WM, Tuinier S. Prader-Willi syndrome: atypical psychoses and motor dysfunctions. Int Rev Neurobiol 2006:72:119-30.

14. Curfs LMG, Fryns JP. Prader-Willi syndrome: a review with special attention to cognitive and behavioral profile. Birth Defects 1992;28:99-104.

15. Einfeld SL, Kavanagh SJ, Smith A, Evans EJ, Tonge BJ, Taffe J. Mortality in Prader-Willi syndrome. Am J Ment Retard 2006;111(3):193-8.

16. Holland AJ, Treasure J, Coskeran P, Dallow J. Characteristics of the eating disorder in Prader-Willi syndrome: implications for treatment. J Intellect Disabil Res 1995;39:373-81.

17. Lindgren AC, Barkeling B, Hagg A, Ritzen EM, Marcus C Rossner S. Eating behavior in Prader-Willi syndrome, normal weight, and obese control groups. J Pediatr 2000;137:50-5. 
18. Hinton EC, Holland AJ, Gellatly MS, Soni S, Patterson M, Ghatei MA, et al. Neural representations of hunger and satiety in Prader-Willi syndrome. Int J Obes 2006;30(2):313-21.

19. Shapira NA, Lessig MC, He AG, James GA, Driscoll DJ, Liu Y. Satiety dysfunction in Prader-Willi syndrome demonstrated by fMRI. J Neurol Neurosurg Psychiatry 2005;76(2):2602.

20. Swaab DF, Purba JS, Hofman MA. Alterations in the hypothalamic paraventricular nucleus and its oxytocin neurons (putative satiety cells) in Prader-Willi syndrome. A study of five cases. J Clin Endocrinol Metab 1995;80:573-9.

21. Cummings DE, Clement $K$, Purnell JO, Vaisse C, Foster KE, Frayo RS, et al. Elevated plasma ghrelin levels in Prader-Willi syndrome. Nat Med 2002;8(7):643-4.

22. Paik KH, Jin DK, Song SY, Lee JE, Ko SH, Song SM, et al. Correlation between fasting plasma ghrelin levels and age, body mass index (BMI), BMI percentiles, and 24-hour plasma ghrelin profiles in Prader-Willi syndrome. J Clin Endocrinol Metab 2004:89(8):3885-9.

23. Tan TM, Vanderpump M, Khoo B, Patterson M, Ghatei MA Goldstone AP. Somatostatin infusion lowers plasma ghrelin without reducing appetite in adults with Prader-Willi syndrome. J Clin Endocrinol Metab 2004;89(8):4162-5.

24. Obata K, Sakazume S, Yoshino A, Murakami N, Sakuta R. Effects of 5 years growth hormone treatment in patients with Prader-Willi syndrome. J Pediatr Endocrinol Metab 2003;16(2):155-62.

25. Hoybye C, Hilding A, Jacobsson H, Thoren M. Growth hormone treatment improves body composition in adults with Prader-Willi syndrome. Clin Endocrinol 2003:58(5):653-61.

26. Hoybye C, Barkeling B, Espelund U, Petersson M, Thoren M. Peptides associated with hyperphagia in adults with PraderWilli syndrome before and during $\mathrm{GH}$ treatment. Growth Horm IGF Res 2003;13(6):322-7.

27. L'Allemand D, Eiholzer U, Schlumpf M, Torresani T, Girard J. Carbohydrate metabolism is not impaired after 3 years of growth hormone therapy in children with Prader-Willi syndrome. Horm Res 2003;59(5):239-48.

28. Eiholzer U, Nordmann Y, L'Allemand D. Fatal outcome of sleep apnoea in PWS during the initial phase of growth hormone treatment. A case report. Horm Res 2002;58(suppl 3):24-6.

29. Sacco M, Di Giorgio G. Sudden death in Prader-Willi syndrome during growth hormone therapy. Horm Res 2005;63(1):29-32.

30. Grugni G, Livieri C, Corrias A, Sartorio A, Crino A; Genetic Obesity Study Group of the Italian Society of Pediatric Endocrinology and Diabetology. Death during GH therapy in children with Prader-Willi syndrome: description of two new cases. J Endocrinol Invest 2005;28(6):554-7.

31. Riedl S, Blumel P, Zwiauer K, Frisch H. Death in two female Prader-Willi syndrome patients during the early phase of growth hormone treatment. Acta Paediatr 2005;94(7):9747.

32. Eiholzer U. Deaths in children with Prader-Willi syndrome. A contribution to the debate about the safety of growth hormone treatment in children with PWS. Horm Res 2005;63(1):33-9.

33. Tata AL, Kockler DR. Topiramate for binge-eating disorder associated with obesity. Ann Pharmacother 2006;40(11):1993-7.

34. Richard D, Ferland J, Lalonde J, Samson P, Deshaies Y. Influence of topiramate in the regulation of energy balance. Nutrition 2000;16:961-6.
35. Smathers SA, Wilson JG, Nigro MA. Topiramate effectiveness in Prader-Willi syndrome. Pediatr Neurol 2003;28(2):130-3.

36. Shapira NA, Lessig MC, Lewis MH, Goodman WK, Driscoll DJ. Effects of topiramate in adults with Prader-Willi syndrome. Am J Ment Retard 2004;109(4):301-9.

37. Shapira NA, Lessig MC, Murphy TK, Driscoll DJ, Goodman WK. Topiramate attenuates self-injurious behaviour in Prader-Willi syndrome. Int J Neuropsychopharmacol 2002;5:141-5.

38. Azoubel VS, Lerário AM, Gomes LG, Cercato C, Villares SMF, Halpern A. Topiramato: uma alternativa para o tratamento de pacientes portadores da síndrome de Prader-Willi. Arq Bras Endocrinol Metab 2003;47(4 supl. 1):S410.

39. Inoue S. Clinical studies with mazindol. Obes Res 1995;3(suppl. 4):549S-52.

40. Itoh M, Koeda T, Ohno K, Takeshita K. Effects of mazindol in two patients with Prader-Willi syndrome. Pediatr Neurol 1995;13(4):349-51.

41. Inui A, Uemoto M, Takamiya S, Shibuya Y, Baba S, Kasuga M. A case of Prader-Willi syndrome with long-term mazindol treatment. Arch Intern Med 1997;157(4):464.

42. Mancini MC, Halpern A. Pharmacological treatment of obesity. Arq Bras Endocrinol Metab 2006;50(2):377-89.

43. Dech $B$, Budow $L$. The use of fluoxetine in an adolescent with Prader-Willi syndrome. J Am Acad Child Adolesc Psychiatry 1991;30(2):298-302.

44. Warnock JK, Kestenbaum T. Pharmacologic treatment of severe skin-picking behaviors in Prader-Willi syndrome. Two case reports. Arch Dermatol 1992;128(12):1623-5.

45. Benjamin E, Buot-Smith T. Naltrexone and fluoxetine in Prader-Willi syndrome. J Am Acad Child Adolesc Psychiatry 1993;32(4):870-3.

46. Hellings JA, Warnock JK. Self-injurious behavior and serotonin in Prader-Willi syndrome. Psychopharmacol Bull 1994;30(2):245-50.

47. Schepis C, Failla $P$, Siragusa M, Palazzo R, Romano C. Failure of fluoxetine to modify the skin-picking behaviour of PraderWilli syndrome. Australas J Dermatol 1998;39(1):57-8.

48. Kohn Y, Weizman A, Apter A. Aggravation of food-related behavior in an adolescent with Prader-Willi syndrome treated with fluvoxamine and fluoxetine. Int J Eat Disord 2001:30(1):113-7.

49. Paraíba DB, Tomé F, Cercato C, Villares SMF, Halpern A. Perda de peso efetiva com uso de fluoxetina em pacientes com síndrome de Prader-Willi: relato de caso. Arq Bras Endocrinol Metab 2002;46(4 supl. 1):S505.

50. Dousei T, Miyata M, Izukura M, Harada T, Kitagawa T, Matsuda $H$. Long-term follow-up of gastroplasty in a patient with Prader-Willi syndrome. Obes Surg 1992;2:189-93.

51. de Almeida MQ, Cercato C, Rascovski A, Goldbaum TS, de Figueiredo DA, Matsuda M, et al. Results of biliopancreatic diversion in two patients with Prader-Willi syndrome. Obes Surg 2005;15(6):901-4.

52. Scopinaro N. Biliopancreatic diversion: mechanisms of action and long-term results. Obes Surg 2006;16(6):683-9.

\section{Endereço para correspondência:}

Daniel F. de Carvalho

Av. Dr. Enéas de Carvalho Aguiar 255, 5andar, bloco 4B, sala $11-\mathrm{A}$

05403-000 São Paulo, SP 